\title{
Multidisciplinary therapy for the treatment of malocclusion in a patient with chronic periodontitis with a five-year follow-up: A case report
}

\author{
DONGMEI ZHANG ${ }^{1}$, LI LIN $^{1}$, XIAOLING TANG ${ }^{1}$, CHEN LI $^{1}$, \\ JINGBO LIU $^{1}$, HONGYANG WANG ${ }^{1,2}$ and YAPING PAN ${ }^{1}$ \\ ${ }^{1}$ Department of Periodontics and Oral Biology, School of Stomatology, China Medical University, Shenyang, \\ Liaoning 110002, P.R. China; ${ }^{2}$ Department of Medicine, University of Virginia, Charlottesville, VA 22908, USA
}

Received September 20, 2016; Accepted June 15, 2017

DOI: $10.3892 /$ etm.2017.4885

\begin{abstract}
Multidisciplinary therapy is essential in dental practice to achieve optimized outcomes. The present case report describes the application of periodontal surgery with a five-year follow-up in a patient with malocclusion and chronic periodontitis. In the presence of periodontal inflammation, orthodontic therapy may result in further periodontal breakdown due to plaque accumulation. In order to prevent this progression, scaling and root planning with a periodontal endoscope was applied, and continuous clinical monitoring and risk assessment was performed every 3 months using a Florida Probe. This combined treatment supports the long-term maintenance of periodontal conditions, functional occlusion and harmony of the facial profile.
\end{abstract}

\section{Introduction}

Periodontitis is a ubiquitous disease affecting $>50 \%$ of the adult population worldwide (1), and is the primary cause of tooth extraction in adults. It is associated with various systemic diseases (2), including cardiovascular disease, diabetes, rheumatoid arthritis, preeclampsia, preterm birth and inflammatory bowel disease $(3,4)$.

A healthy periodontal condition is the bedrock of oral health, and successful periodontal management remains essential for the success of other oral therapies. Evidently, considering that multidisciplinary treatment is an integral part of dental practice, collaboration among dental team members is vital in order to meet the growing esthetic demands of patients (5).

Correspondence to: Dr Yaping Pan, Department of Periodontics and Oral Biology, School of Stomatology, China Medical University, 117 Nanjing North Street, Shenyang, Liaoning 110002, P.R. China E-mail: yppan@mail.cmu.edu.cn

Key words: chronic periodontitis, multidisciplinary therapy, orthognathic surgery, periodontal maintenance therapy
It is hypothesized that orthodontics in the presence of inflammation may lead to rapid and irreversible breakdown of the periodontium. Orthodontic treatment is usually contraindicated in patients with active periodontal disease or poor periodontal health (6). Therefore, impeccable periodontal maintenance therapy (PMT) is important for prevention of disease progression during tooth movement. The present case report demonstrates a five-year follow-up of a patient with chronic periodontitis who underwent multidisciplinary therapy, including periodontal initial therapy, orthodontic treatment, orthognathic surgery and a series of PMTs.

\section{Case report}

Clinical observations. A systemically healthy 24-year-old female who came to the orthodontic office of Stomatological Hospital, China Medical University (Shenyang, China) for esthetic management in August 2010, was then referred to a periodontal clinic for evaluation and treatment. In the anamnesis, she complained of gum bleeding while brushing, oral malodor and food impaction for the preceding 2 years. Examination (Fig. 1) revealed osseous reverse occlusion, maxillary construction and mandibular prognathism as well as chronic periodontitis (Fig. 1A). The patient exhibited very poor plaque control, with $100 \%$ plaque accumulation. Furthermore, the probing depth (PD) was between 1 and $5 \mathrm{~mm}$ (the percentage of sites with $\mathrm{PD} \geq 5 \mathrm{~mm}$ was $4.1 \%$, which was predominantly distributed at the proximal surface teeth no. 15 and 16 and the percentage of sites with $5>\mathrm{PD} \geq 4 \mathrm{~mm}$ was $24.7 \%$ ). Additionally, the average clinical attachment loss was 2.4, positive bleeding on probing (BOP) sites was $41.7 \%$ and the risk assessment level was high, as detected by a Florida Probe (FP32, version 6; Florida Probe Corporation, Gainesville, FL, USA; Fig. 1C and D). The intraoral radiographic views demonstrated mild destruction of the alveolar bone, which was observed in the angular absorption of the alveolar bone in the mesial surface of teeth no. 16, 23, 26, 47, as well as the distal surface of teeth no. 16 and 37 (Fig. 1B).

According to the diagnostic standards of Armitage et al (7), the patient was diagnosed with the following: i) Moderate chronic periodontitis, ii) Angle Class III skeletal malocclusion, 
A

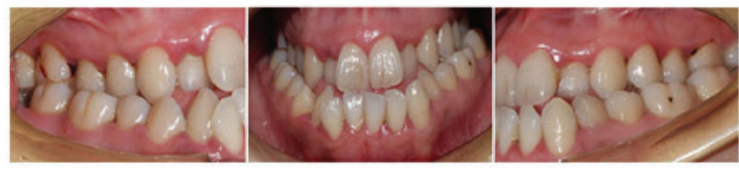

B

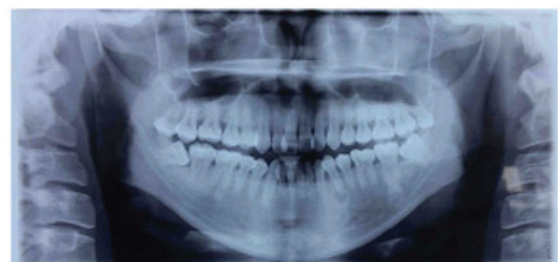

C

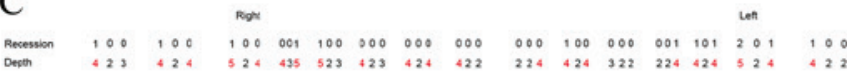
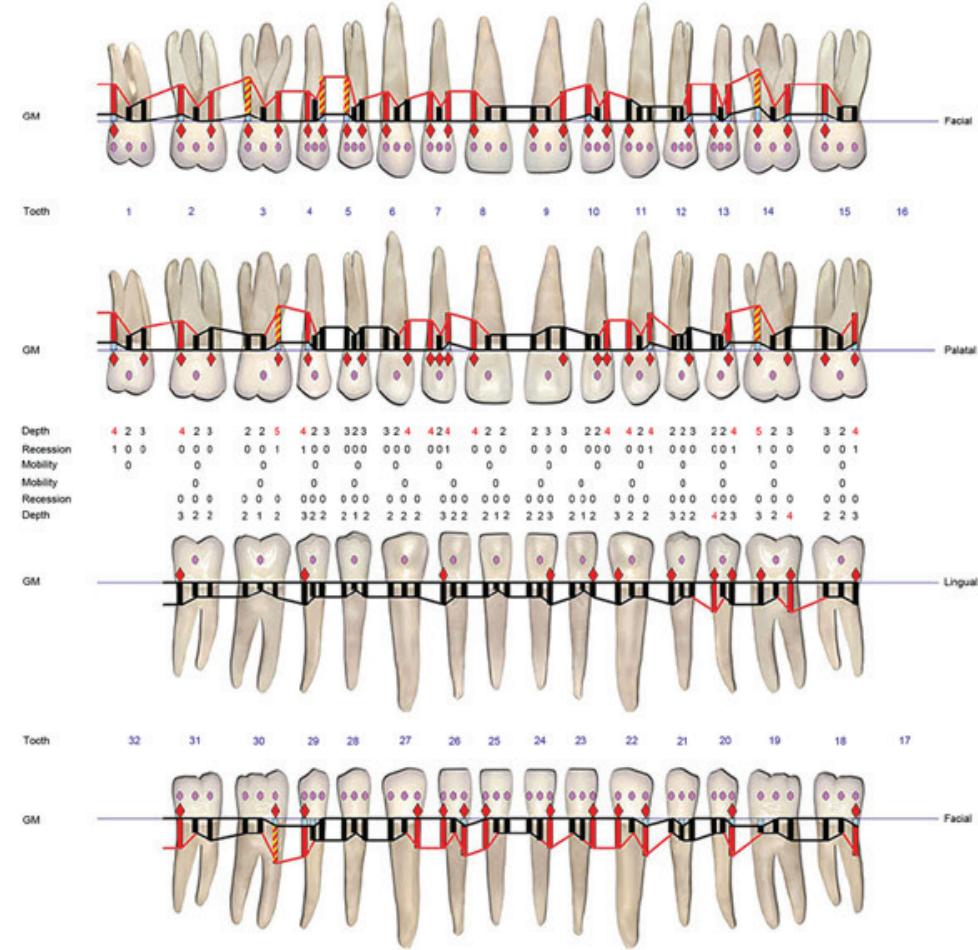

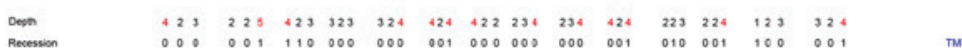

$\mathrm{D}$

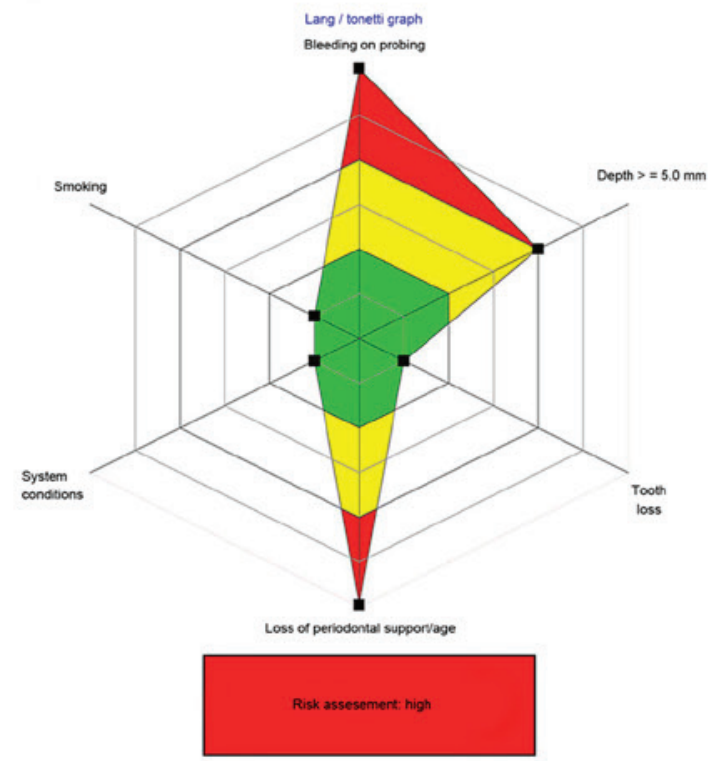

Figure 1. (A) Intraoral images and (B) radiographic views prior to treatment exhibiting mild to moderate inflammation and destruction of the alveolar bone, particularly in the molar region. (C) Periodontal chart and (D) risk assessment by Florida Probe prior to treatment. Periodontal measurement prior to periodontal initial therapy. GM, gingival margin. 
A

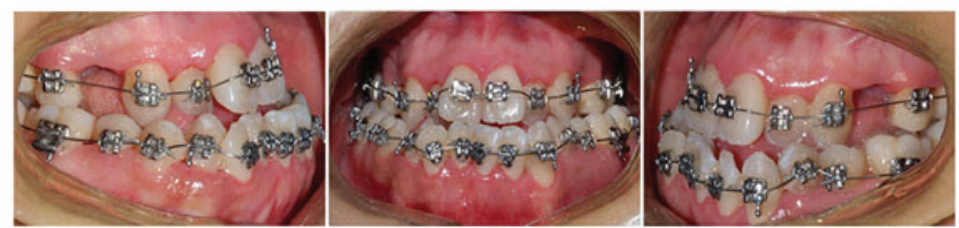

B

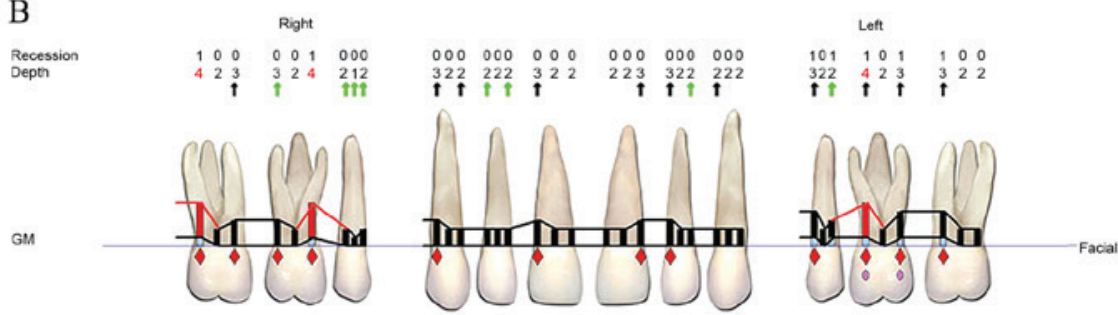

Tooth

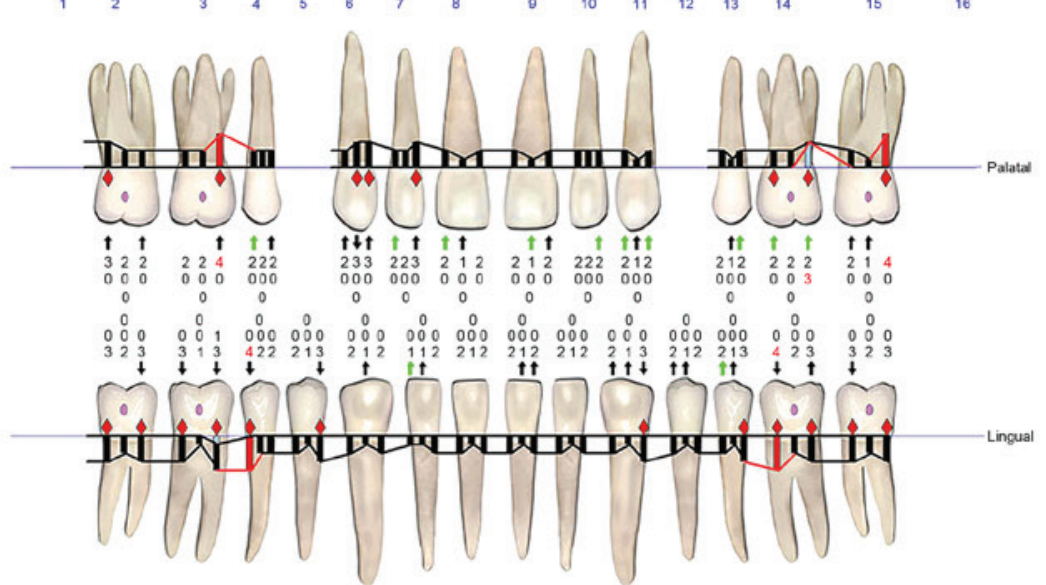

Toon

GM

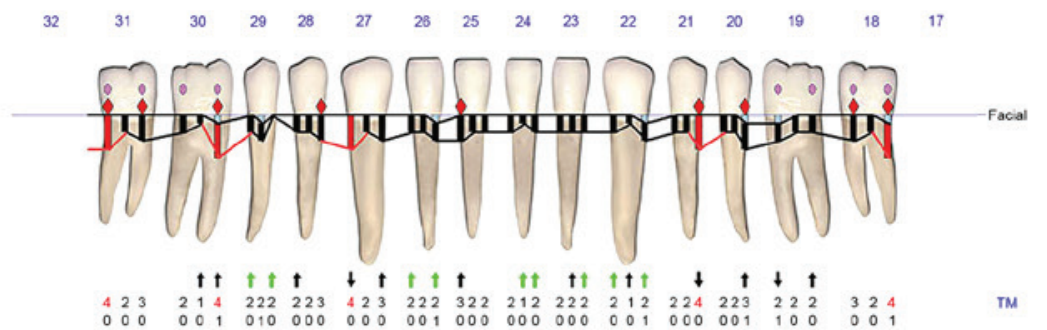

Dept
Recession

$\begin{array}{llllllllllllllllllllllll}4 & 2 & 3 & 2 & 1 & 4 & 222 & 223 & 4 & 23 & 222 & 322 & 212 & 222 & 2 & 1 & 224 & 223 & 2 & 2 & 2 & 3 & 2 \\ 0 & 0 & 0 & 0 & 0 & 1 & 010 & 000 & 0 & 0 & 0 & 001 & 000 & 000 & 000 & 0 & 0 & 0 & 00 & 001 & 10 & 0 & 0 & 0\end{array}$

$\mathrm{C}$
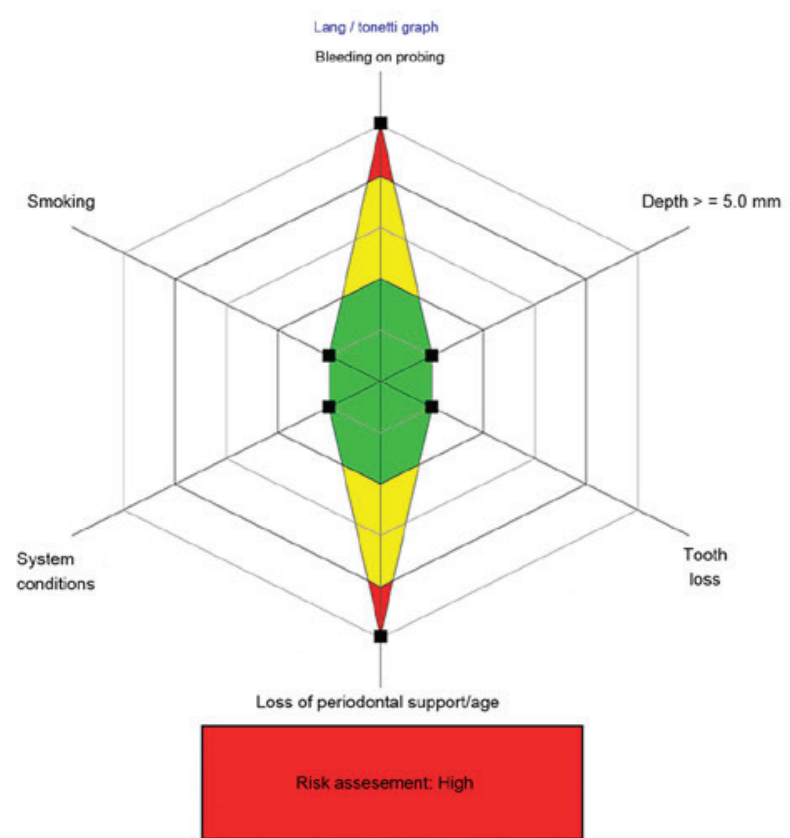

Figure 2. (A) Intraoral images, (B) periodontal chart and (C) risk assessment by Florida Probe following the periodontal initial therapy. Inflammation was controlled, positive BOP sites and plaque accumulation was evidently decreased while orthodontic treatment was ongoing. GM, gingival margin. 
maxillary retrusion and mandibular prognathism and iii) moderate crowded malocclusion. Furthermore, the treatment plan was proposed by a team of periodontists, orthodontists and plastic surgeons. The patient accepted the treatment plan, and therapy consent was acquired. Furthermore, the patient provided written informed consent for their inclusion in the present case report, and the report was reviewed and approved by the Ethics Committee of the School of Stomatology, China Medical University (Shenyang, China).

Treatment goals. Goals of the treatment plan were as follows: i) Eliminate ongoing periodontal infection, ii) reestablish the functional and esthetic situation by orthodontic and surgical operation, and iii) achieve optimal occlusal relationships.

Treatment plan. The treatment plan included: i) A periodontal initial therapy; ii) presurgical orthodontic treatment; iii) orthognathic surgery, consisting of Le-Forte I osteotomy of maxilla and maxillary advancement and bilateral sagittal split osteotomy for mandibular setback; iv) postsurgical orthodontic treatment; v) a long-term retention plan and vi) continuous PMT.

Periodontal initial therapy. Periodontal initial therapy is based on mechanical removal of bacterial plaque via non-surgical treatment. Standardized supra- and subgingival scaling and root planning (SRP) was applied. To achieve total calculus removal, SRP with a periodontal endoscope was implemented in the sites with pockets $\geq 5 \mathrm{~mm}$. At 2 weeks following this procedure, the patient underwent a session of professional plaque control, including reinforcement of an oral hygiene process. Additionally, 12 weeks after completion of the active therapy, periodontal inflammation was controlled. Positive BOP sites were reduced from 41.7-26.2\%, and plaque accumulation was markedly decreased from $100.0-17.4 \%$, with the mean PD being $2.2 \mathrm{~mm}$. Therefore, orthodontic treatment was initiated. Furthermore, a thorough examination and risk assessment were performed using a Florida Probe as demonstrated in Fig. 2. The PD was markedly improved, however the risk assessment level remained high due to the orthodontic appliance. The treatment continued to presurgical orthodontics. Furthermore, the patient was recalled for periodontal maintenance every 3 months throughout the orthodontic therapy and during these visits the dentists examined the periodontal parameters and recorded any change in the patient's medical condition. Finally, supragingival prophylaxis was performed.

Presurgical orthodontic treatment. In order to facilitate the proper positioning of the jaws during surgery, presurgical orthodontic treatment was performed to initially align the teeth, followed by straightening of the mandibular anterior teeth. Briefly, the first premolars and third molar of the maxilla were removed, nickel titanium wires were used for alignment of the arches and class II elastics were used for correction of the incisor position. Finally, the duration of presurgical orthodontics was $\sim 18$ months.

Orthognathic surgery. Le-Forte I surgery and bilateral sagittal split osteotomy were performed due to its potential to place maxilla superiorly and forwardly, which was able to correct the

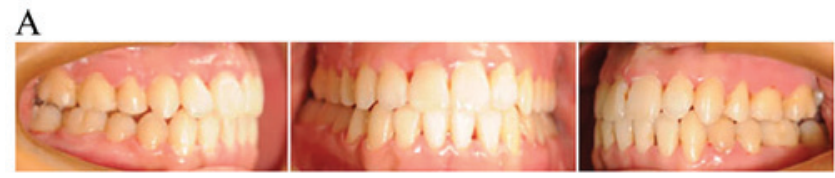

B

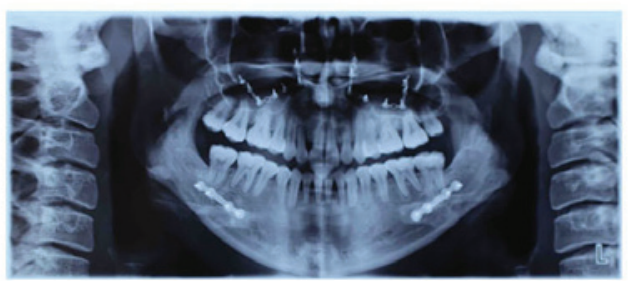

Figure 3. (A) Intraoral images and (B) radiographic views at 3 years post-treatment. The esthetic profile was enhanced and the radiographic bone density was increased in the molar region following periodontal, orthodontic and surgery treatment. Note the stable outcome following the multidisciplinary treatment.

class III skeletal malocclusion along with the bilateral sagittal split osteotomy with the mandibular drawing back. The jaws were repositioned as decided by research models and occlusal guide plates. The maxilla and mandible were splinted together to gain the fixation and the surgery was then successfully performed without any complications. The temporomandibular joint was intact and no manifestations of temporomandibular disorders followed.

Postsurgical orthodontic treatment and long-term retention. Focusing on the maintenance of the jaw relationship and meticulous adjustment of occlusion, postsurgical orthodontic treatment was accomplished within 5 months, beginning at 4 weeks post-surgery. Functional occlusion and an improved facial profile were achieved following the surgical and orthodontic treatments. For a long-term retention plan, bimaxillary Hawley-type of retainers were provided to wear full time in order to prevent recurrence of malocclusion.

$P M T$. It is noteworthy that PMT was performed throughout the multidisciplinary treatment process. In particular, the patient was scheduled to re-visit the periodontist every 3 months during the orthodontic and surgical treatment. The patient's facial profiles in clinical and radiographic improvement following 3 years of post-treatment are demonstrated in Fig. 3. Periodontal parameters and risk assessments were obtained by a Florida Probe as demonstrated in Fig. 4. The PD was stable and positive BOP sites were continuously decreased to $14.1 \%$. Although the risk assessment level was moderate, it is of paramount importance that patients still require continuous clinical monitoring, even after periodontal treatment. Subsequently, a five-year visit was recorded and demonstrated in Fig. 5. Compared with the view of pretreatment. Periodontal parameters and changes prior to and following multidisciplinary therapy are provided in Table I.

\section{Discussion}

Skeletal class III anomalies are one of the most complex problems in childhood and adulthood dentofacial abnormalities. Patients' demands for improved esthetics have led to notable 


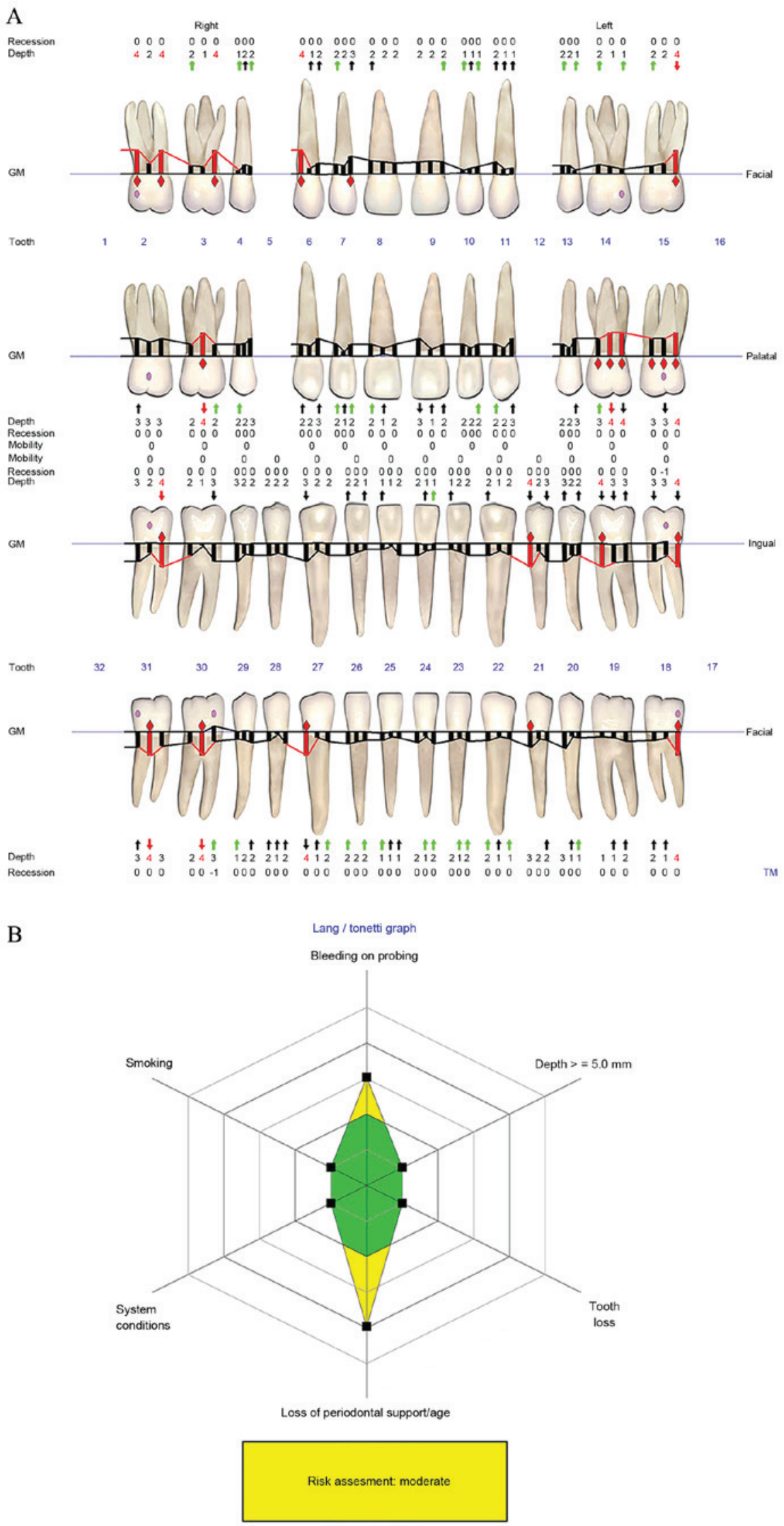

Figure 4. (A) Periodontal chart and (B) risk assessment by Florida Probe at 3 years post-treatment. Periodontal parameters were stable and the risk assessment level was moderate since the completion of the orthodontal surgery process. GM, gingival margin.

changes in the field of dentistry (8). Although orthodontic treatment does not lead to periodontal attachment loss (9), in the presence of periodontal inflammation orthodontic movement may result in further uncontrolled periodontal 
Table I. Periodontal parameters prior and post-periodontal initial therapy.

\begin{tabular}{llll}
\hline Clinical assessment & Prior to treatment $(\%)$ & 3 months post-treatment $(\%)$ & 3 years post-treatment $(\%)$ \\
\hline
\end{tabular}

\section{PD}

$\begin{array}{lr}\mathrm{PD} \geq 5 \mathrm{~mm} & 4.1 \\ 4 \leq \mathrm{PD}<5 \mathrm{~mm} & 24.7 \\ \mathrm{PD}>4 \mathrm{~mm} & 71.2 \\ \text { BOP-positive } & 41.7 \\ \text { Plaque-positive } & 100.0\end{array}$

0.0

7.7

92.3

26.2

17.4
0.0

10.9

89.1

14.1

8.7

PD, probing depth; BOP, bleeding on probing.

A

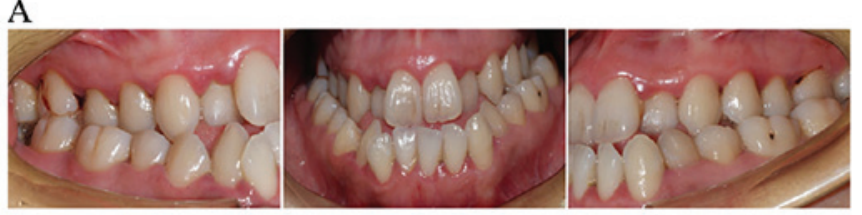

B

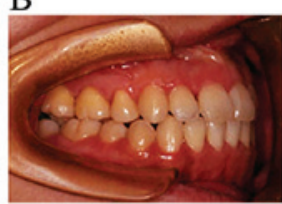

C

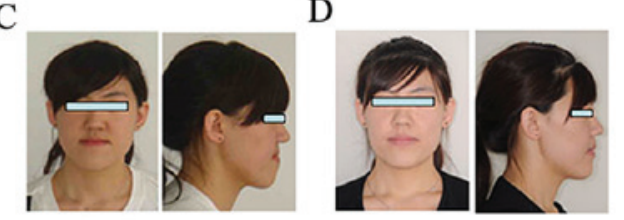

Figure 5. (A and B) Intraoral and (C and D) exoral images (A and C) prior to and $(\mathrm{B}$ and $\mathrm{D})$ at 5 years following multidisciplinary treatment. Comparative views of significant improvement in dentition, occlusion and esthetic facial profile. Note the maintenance of healthy periodontal conditions.

breakdown. Furthermore, Takai et al (10) have suggested that surgical procedure at infected sites is more likely to produce bacteremia. Therefore, inflammation must be controlled prior to initiating orthodontic surgery therapy.

The aim of periodontal therapy is to resolve inflammation and prevent disease progression (11). The primary treatment of chronic periodontitis is based on removal of bacterial plaque via non-surgical therapy, which was considered the gold standard of periodontal therapy $(12,13)$. It has been determined that periodontal endoscopy demonstrates an improvement in calculus removal with minimal invasion (14). Therapeutic outcomes revealed improvements in clinical parameters. Following SRP with periodontal endoscopy, the percentage of sites with $\mathrm{PD} \geq 4 \mathrm{~mm}$ decreased from $24.7-7.7 \%$, and the percentage of positive BOP sites was reduced from 41.7-26.2\%. Considering the orthodontic appliances that were used, the result of the treatment was acceptable. In addition, following orthodontics and surgery treatment, the outcome revealed that the improvement of parameters continues, with mean PD values observed between 2.2 and $2.1 \mathrm{~mm}$ and positive BOP sites from 26.2-14.1\%. The results indicate that surgical-orthodontic periodontal treatment is effective for improving skeletal disharmony, facial profile and periodontal condition.

PMT is considered to be an indispensable component of periodontal therapy, which has a notable impact on periodontal prognosis (14). The long-term maintenance of the therapeutic results was a challenge for clinicians and patients in this case. Therefore, 3 months later (following the active periodontal therapy) reassessment was performed. The patient's risk assessment level remained high due to the orthodontic appliance. It has recently been shown that in high-risk patients the critical threshold for plaque accumulation to trigger periodontitis is low (2). Furthermore, it is accepted that maintenance programs should be implemented to minimize or neutralize the influence of risk determinants (15). Therefore, continuous clinical monitoring was performed and recall visits were designed to be carried out at 3-month intervals throughout the multidisciplinary treatment program. Additionally, reinforcement of oral hygiene is essential during every visit. Most importantly, successful treatment necessitates behavior change in patients to attain and sustain high standards of daily plaque removal. Therefore, an oral irrigator and orthodontic toothbrush were recommended for improved plaque control, particularly in the interproximal area beneath the orthodontic wires. It is also noteworthy that the high-risk profile of the patient at baseline was subsequently changed to a moderate risk profile, reflecting the non-recurrence of periodontitis, and the preservation of alveolar bone

The aim of the present case report was to treat a patient with class III skeletal malocclusion, crowed malocclusion and chronic periodontitis using multidisciplinary treatment in combination with systematic periodontal supportive therapy. This long-term case report demonstrated that periodontal therapy with orthodontics and orthognathic surgery, yielded content reductions in PDs and radiographic bony fill, and sustained periodontal results. In addition, a favorable outcome, including functional occlusion and a harmonious facial profile were achieved following this multidisciplinary therapy.

\section{Acknowledgements}

The authors of the present study would like to thank Li Lu, Zhiming Hou and Xiaofeng Bai for assisting in the preparation of the manuscript. Funding from Liaoning Clinical Capability Construction Programs of Provincial Health and Family Planning Commission (grant no. LNCCC-C08-2015) is gratefully acknowledged. 


\section{References}

1. Petersen PE and Ogawa H: The global burden of periodontal disease: Towards integration with chronic disease prevention and control. Periodontol 2000 60: 15-39, 2012.

2. Chapple IL, Van der Weijden F, Doerfer C, Herrera D, Shapira L, Polak D, Madianos P, Louropoulou A, Machtei E, Donos N, et al: Primary prevention of periodontitis: Managing gingivitis. J Clin Periodontol 42 (Suppl 16): S71-S76, 2015.

3. Badran Z, Struillou X, Verner C, Clee T, Rakic M, Martinez MC and Soueidan A: Periodontitis as a risk factor for systemic disease: Are microparticles the missing link? Med Hypotheses 84: 555-556, 2015.

4. Cullinan MP and Seymour GJ: Periodontal disease and systemic illness: Will the evidence ever be enough? Periodontol 200062 : 271-286, 2013

5. Kim YI, Kim MJ, Choi JI and Park SB: A multidisciplinary treatment for the management of pathologic tooth migration in a patient with moderately advanced periodontal disease. Int J Periodontics Restorative Dent 32: 225-230, 2012.

6. Alfuriji S, Alhazmi N, Alhamlan N, Al-Ehaideb A, Alruwaithi M, Alkatheeri $\mathrm{N}$ and Geevarghese $\mathrm{A}$ : The effect of orthodontic therapy on periodontal health: A review of the literature. Int J Dent 2014: 585048, 2014

7. Armitage GC, Wu Y, Wang HY, Sorrell J, di Giovine FS and Duff GW: Low prevalence of a periodontitis-associated interleukin-1 composite genotype in individuals of Chinese heritage. J Periodontol 71: 164-171, 2000.
8. Pinto RC, Chambrone L, Colombini BL, Ishikiriama SK, Britto IM and Romito GA: Minimally invasive esthetic therapy: A case report describing the advantages of a multidisciplinary treatment. Quintessence Int 44: 385-391, 2013.

9. Re S, Corrente G, Abundo R and Cardaropoli D: Orthodontic treatment in periodontally compromised patients: 12 -year report. Int J Periodontics Restorative Dent 20: 31-39, 2000.

10. Takai S, Kuriyama T, Yanagisawa M, Nakagawa K and Karasawa T: Incidence and bacteriology of bacteremia associated with various oral and maxillofacial surgical procedures. Oral Surg Oral Med Oral Pathol Oral Radiol Endod 99: 292-298, 2005.

11. Farman M and Joshi RI: Full-mouth treatment versus quadrant root surface debridement in the treatment of chronic periodontitis: A systematic review. Br Dent J 205: E18; 496-497, 2008.

12. Baelum V and López R: Defining and predicting outcomes of non-surgical periodontal treatment: A 1-yr follow-up study. Eur J Oral Sci 124: 33-44, 2016.

13. Cobb CM: Clinical significance of non-surgical periodontal therapy: An evidence-based perspective of scaling and root planing. J Clin Periodontol 29 (Suppl 2): S6-S16, 2002.

14. Montevecchi M, De Blasi V and Checchi L: Is implant flossing a risk-free procedure? A case report with a 6-year follow-up. Int J Oral Maxillofac Implants 31: e79-e83, 2016.

15. Lorentz TC, Cota LO, Cortelli JR, Vargas AM and Costa FO: Prospective study of complier individuals under periodontal maintenance therapy: Analysis of clinical periodontal parameters, risk predictors and the progression of periodontitis. J Clin Periodontol 36: 58-67, 2009. 